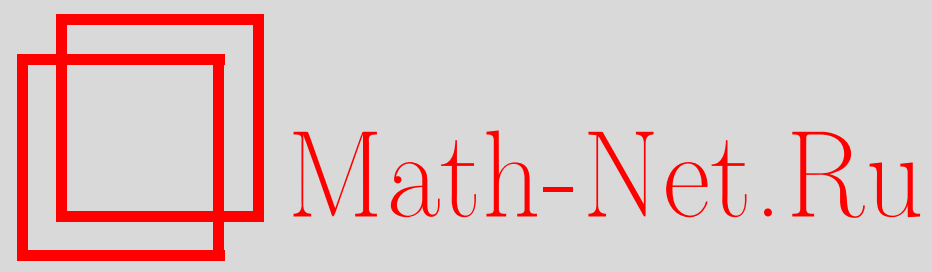

А. Н. Черепов, Приближение непрерывных функций конечными автоматами, Дискрет. матем., 2012, том 24, выпуск $3,82-89$

DOI: https://doi.org/10.4213/dm1200

Использование Общероссийского математического портала Math-Net.Ru подразумевает, что вы прочитали и согласны с пользовательским соглашением http://www . mathnet.ru/rus/agreement

Параметры загрузки:

IP : 44.207 .124 .84

26 апреля 2023 г., 13:37:50 
УДК 519.95

\title{
Приближение непрерывных функций конечными автоматами
}

\author{
() 2012 г. А. Н. Черепов
}

\begin{abstract}
Рассматриваются конечные автоматы с задержкой, являющиеся естественным обобщением конечных автоматов. Ставится задача приближения непрерывных функций автоматами с задержкой и доказано утверждение о возможности приближения с произвольной точностью любой непрерывной функции. Приведены приближения некоторых функций и даны оценки числа их состояний. Для нескольких классов непрерывных функций приводятся оценки числа состояний автоматов, $\varepsilon$-приближающих функции этих классов.
\end{abstract}

\section{1. Детерминированные функции с задержкой и конечные автоматы с задержкой}

Рассмотрим множество всех бесконечных двоичных последовательностей $E$. Множество всех функций вида $f: E^{n} \rightarrow E$ обозначим $P$. Предположим, что $a_{1}, a_{2}, \ldots, a_{n}-$ последовательности из $E$, а $\tilde{a}=\left(a_{1}, a_{2}, \ldots, a_{n}\right)$ - набор таких последовательностей. Пусть $a_{1}\left|k, a_{2}\right| k, \ldots, a_{n} \mid k$ - первые $k$ членов последовательностей $a_{1}, a_{2}, \ldots, a_{n}$ соответственно, тогда $\tilde{a} \mid k=\left(a_{1}\left|k, a_{2}\right| k, \ldots, a_{n} \mid k\right)$.

Назовем функцию $f$ детерминированной, если для всех $k=1,2, \ldots$ и для любых $\tilde{a}, \tilde{b}$ соотношение $\tilde{a}|k=\tilde{b}| k$ влечет $f(\tilde{a})|k=f(\tilde{b})| k$. Класс всех детерминированных функций обозначим $P_{d}$; все остальные функции из $P$ будем называть недетерминированными.

Определение $1([4,5])$. Говорим, что функция $f$ является детерминированной функцией с задержкой $\tau$, где $\tau$ - произвольное неотрицательное целое число, если для любого $k=1,2,3, \ldots$ и любых $\tilde{a}, \tilde{b}$ соотношение $\tilde{a}|k+\tau=\tilde{b}| k+\tau$ влечет $f(\tilde{a})|k=f(\tilde{b})| k$.

Множество детерминированных функций с задержкой можно определить и следующим образом.

Определение 2. Говорим, что функция $f$ является детерминированной функцией с задержкой $\tau$, где $\tau$ - произвольное неотрицательное целое число, если существует такая детерминированная функция $g$, что для любого $\tilde{a}$ и $b=g(\tilde{a}), b=b(1) b(2) b(3) \ldots$ значение функции $f$ на наборе $\tilde{a}$ равно $f(\tilde{a})=b(\tau+1) b(\tau+2) b(\tau+3) \ldots$

Лемма $1([4,5])$. Определения 1 и 2 эквивалентны.

Из определения 2 следует, что функцию с задержкой $\tau$ от $n$ аргументов можно трактовать так: берется дискретное детерминированное устройство с $n$ входами, преобразующее 
бесконечные входные последовательности из нулей и единиц в такую же выходную последовательность, и рассматривается выход этого устройства не с первого момента времени, а с момента времени $\tau+1$. Осуществляемое преобразование и считается детерминированной функцией с задержкой $\tau$. Таким образом, понятие функции с задержкой являются естественным обобщением детерминированных функций. Аналогичным образом можно ввести понятие конечного автомата с задержкой. Будем рассматривать инициальные автоматы с $n$ входами и одним выходом, преобразующие бесконечные двоичные последовательности, то есть реализующие дискретные функции из множества $P$.

Определение 3. Назовем конечным автоматом с задержкой $\tau$ конечный автомат, который при подаче на его входы некоторых входных последовательностей начинает формировать выходную последовательность не с первого момента времени, а с некоторой задержкой, не превосходящей $\tau$.

Отличие автоматов с задержкой от обычных конечных автоматов удобно проиллюстрировать на диаграмме переходов. Если обычный автомат начинает формировать разряды выхода, начиная с первого такта работы, то автомат с задержкой $\tau$ для некоторых входных последовательностей в течении нескольких (не более $\tau$ ) тактов работы не формирует выход, а только переходит из одного состояния в следующее. Затем конечный автомат с задержкой начинает функционировать как обычный конечный автомат. Очевидно, что каждую детерминированную функцию с задержкой $\tau$ можно реализовать некоторым конечным автоматом с задержкой $\tau$.

Перейдем к вопросу о возможности реализации непрерывных функций, осуществляющих отображение точек $n$-мерного единичного куба на отрезок $[0,1]$. Сопоставим каждой двоичной последовательности $a(1) a(2) \ldots a(i) \ldots$ некоторое число из отрезка $[0,1]$, равное $0, a(1) a(2) \ldots a(i) \ldots$ Из двух возможных представлений $0, a(1) a(2) \ldots a(i) 100 \ldots=$ $0, a(1) a(2) \ldots a(i) 0111 \ldots$ выберем первое. Для числа 1 берется представление $1=0,1111 \ldots$

Определение 4. Функцию $\mu:[0,1] \rightarrow E$ определим следующим образом: для любого числа $a \in[0,1]$, где $a=0, a_{1} a_{2} a_{3} \ldots$, значение функции равно $\mu(a)=a_{1} a_{2} a_{3} \ldots$

Определение 5. Функцию $\mu^{-1}: E \rightarrow[0,1]$ определим так, что для любой бесконечной двоичной последовательности $a=a_{1} a_{2} a_{3} \ldots$ справедливы следующие утверждения:

(1) если существует натуральное число $k$ такое, что $a=a_{1} a_{2} \ldots a_{k} 01111 \ldots$, то есть, начиная с $(k+2)$-го члена, $a$ представляет собой бесконечную последовательность из единиц, то $\mu^{-1}(a)=0, a_{1} a_{2} \ldots a_{k} 1000 \ldots$;

(2) для остальных последовательностей $\mu^{-1}(a)=0, a_{1} a_{2} a_{3} \ldots$

Определение 6. Любой дискретной функции $f: E^{n} \rightarrow E$ однозначно сопоставим действительную функцию $g:[0,1]^{n} \rightarrow[0,1]$ так, что для любой $n$-мерной точки $\left(b_{1}, b_{2}, \ldots, b_{n}\right) \in[0,1]^{n}$ верно равенство

$$
g\left(b_{1}, b_{2}, \ldots, b_{n}\right)=\mu^{-1} f\left(\mu\left(b_{1}\right), \mu\left(b_{2}\right), \ldots, \mu\left(b_{n}\right)\right) .
$$

Действительную функцию $g$ будем называть функцией, соответствующей дискретной функции $f$.

Заметим, что при таком сопоставлении теряется информация о преобразовании последовательностей вида $a_{1} a_{2} \ldots a_{i} 0111 \ldots$ 
Определение 7. Пусть $\varepsilon>0$; будем говорить, что функция $d(\tilde{x}) \varepsilon$-равна функции $f(\tilde{x})$ на единичном кубе, если при любом $\tilde{x} \in[0,1]^{n}$ выполняется неравенство

$$
|f(\tilde{x})-d(\tilde{x})|<\varepsilon .
$$

Будем также говорить, что в этом случае $d(\tilde{x}) \varepsilon$-приближает $f(\tilde{x})$.

Если функция $d(\tilde{x}) \varepsilon$-приближает $f(\tilde{x})$ и соответствует дискретной функции, реализуемой конечным автоматом $A$ с задержкой $\tau$, то будем говорить, что автомат $A$ $\varepsilon$-приближает $f(\tilde{x})$.

Не всякую непрерывную функцию можно $\varepsilon$-приблизить обычным конечным автоматом, так как не всякую непрерывную функцию можно $\varepsilon$-приблизить детерминированной функцией (см. [4]). Но конечных автоматов с задержкой уже достаточно.

Выберем шаг $h=2^{-k}$ и рассмотрим множество всех точек отрезка $[0,1]$, взятых с этим шагом, вида $x_{i}=i 2^{-k}, i=0,1,2, \ldots, 2^{k}$. Это множество будем называть решеткой точек с шагом $h=2^{-k}$ и обозначать $I_{h}$. Аналогичным образом, в $n$-мерном кубе $[0,1]^{n}$ введем решетку $I_{h}^{n}$ как декартову степень решетки $I_{h}$. Пусть $\tilde{a}=\left(a_{1}, a_{2}, \ldots, a_{n}\right)-$ произвольная точка решетки $I_{h}^{n}$. Интервалом $I_{h}(\tilde{a})$ решетки $I_{h}^{n}$ назовем множество точек $\tilde{x}=\left(x_{1}, x_{2}, \ldots, x_{n}\right) n$-мерного куба $[0,1]^{n}$ таких, что $a_{i} \leqslant x_{i}<h$ при $i=1,2, \ldots$

Теорема 1. Для любого $\varepsilon>0$ и любой функиии $f(\tilde{x}) \in C[0,1]^{n}$ существуют число $\tau \geqslant 0$ $u$ конечный автомат А с задержкой $\tau$ такие, что автомат $A$ в-приближает $f(\tilde{x})$.

Доказательство. Рассмотрим произвольное $\varepsilon>0$. Так как непрерывная на компакте функция является равномерно непрерывной, существует $\delta>0$ такое, что из неравенства $|\tilde{x}-\tilde{y}|<\delta$ следует, что $|f(\tilde{x})-f(\tilde{y})|<\varepsilon / 2$. Выберем $\tau$ таким образом, что $2^{-\tau}<\delta$, и рассмотрим решетку $I_{h}^{n}$ при $h=2^{-\tau}$. Для каждой точки $\tilde{a}_{i}, i=0,1, \ldots$, решетки $I_{h}^{n}$ обозначим через $f_{i}$ значение функции $f$ в этой точке с точностью $\lceil\log (1 / \varepsilon)\rceil+1$ разрядов; здесь и в дальнейшем $\lceil\cdot\rceil$ - целая часть числа с избытком, а логарифм берется по основанию 2. Тогда $f\left(\tilde{a}_{i}\right)-f_{i} \leqslant \varepsilon / 2$ и у числа $f_{i}$, по крайней мере начиная с $\lceil\log (1 / \varepsilon)\rceil+2$ разряда, все разряды равны нулю. Построим конечный автомат $A$ с задержкой $\tau, \varepsilon$-приближающий функцию $f$. Автомат зададим с помощью диаграммы переходов. Для этого рассмотрим двоичное дерево высоты $\tau$, к висячим вершинам которого подвешены обычные конечные автоматы. Каждой ветви двоичного дерева соответствует последовательность состояний автомата с задержкой, которая определяет первые $\tau$ разрядов каждой из точек решетки $I_{h}^{n}$. Корню дерева соответствует начальное состояние и при нахождении в этих состояниях автомат не формирует значения выходов. При этом к висячей вершине, соответствующей первым $\tau$ разрядам точки решетки $\tilde{a}_{i}$, подвесим конечный автомат, реализующей $\lceil\log (1 / \varepsilon)\rceil+1$ первых разрядов числа $f_{i}$, а затем выдающим одни нули. Построенный автомат реализует некоторую дискретную функцию $g(\tilde{x})$ с задержкой $\tau$. Пусть $d(\tilde{x})-$ действительная функция, соответствующая функции $g(\tilde{x})$. Тогда для любого $\tilde{x} \in[0,1]^{n}$ и точки $\tilde{a}_{i}$ решетки $I_{h}^{n}$, совпадающей с $\tilde{x}$ в первых $\tau$ разрядах, справедливо неравенство

$$
\begin{aligned}
|f(\tilde{x})-d(\tilde{x})| & =\left|\left(f(\tilde{x})-f\left(\tilde{a}_{i}\right)\right)+\left(f\left(\tilde{a}_{i}\right)-d(\tilde{x})\right)\right| \\
& \leqslant\left|f(\tilde{x})-f\left(\tilde{a}_{i}\right)\right|+\left|f\left(\tilde{a}_{i}\right)-d(\tilde{x})\right|<\varepsilon,
\end{aligned}
$$

так как

$$
f_{i}=d(\tilde{x}), \quad f\left(\tilde{a}_{i}\right)-f_{i} \leqslant \varepsilon / 2, \quad\left|\tilde{x}-\tilde{a}_{i}\right|<\delta .
$$

Теорема доказана.

Рассмотрим вопросы сложности реализации непрерывных функций конечными автоматами с задержкой. 


\section{2. Оценки сложности приближений непрерывных функций конечными автоматами с задержкой}

Определение 8. Для любой непрерывной функции $f \in C\left([0,1]^{n}\right)$, принимающей значения из отрезка $[0,1]$, и любого $\varepsilon>0$ назовем сложностью реализации в классе конечных автоматов с задержкой $\tau$ наименьшее число состояний автомата, реализующего функцию $f$ с точностью $\varepsilon$.

Сложность реализации функции $f$ будем обозначать $L(f, \varepsilon)$. Пусть задан подкласс $H$ класса непрерывных функций. Положим

$$
L(H, \varepsilon)=\sup \{L(f, \varepsilon): f \in H\} .
$$

В [5] было показано, что функцию $\sqrt[n]{x}$ при любом $\varepsilon=2^{-k}$ можно $\varepsilon$-приблизить детерминированной функцией с задержкой $k(n-1)$. При этом значение задержки является минимальным. Оценим число состояний конечного автомата с задержкой, $\varepsilon$-приближающего эту функцию.

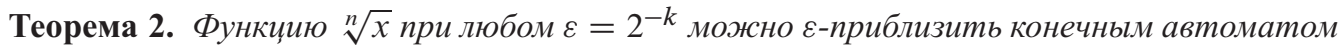
с задержкой, для которого справедлива оценка

$$
L(\sqrt[n]{x}, \varepsilon) \leqslant \sum_{i=0}^{2^{k}-1}\left\lfloor\log \left((i+1)^{n}-i^{n}\right)\right\rfloor+k 2^{k} .
$$

Доказательство. Разобьем отрезок $[0,1]$ на полуинтервалы точками $0,1 / 2^{n k}, 2^{n} / 2^{n k}$, $3^{n} / 2^{n k}, 4^{n} / 2^{n k}, \ldots, i^{n} / 2^{n k},(i+1)^{n} / 2^{n k}, \ldots,\left(2^{k}-1\right)^{n} / 2^{n k}, 1$. Заметим, что изменение функции $\sqrt[n]{x}$ на каждом из полуинтервалов $\left[i^{n} / 2^{n k},(i+1)^{n} / 2^{n k}\right)$ не превосходит $1 / 2^{k}$. Поэтому, если в качестве приближенного значения этой функции взять $k$ первых разряда числа $\sqrt[n]{y}$, где $y$ - середина этого полуинтервала, то это значение будет отличаться от значения этой функции в любой другой точке полуинтервала менее чем на $1 / 2^{k}$. Построим конечный автомат с задержкой, $\varepsilon$-приближающий эту функцию. Автомат состоит из части двоичного дерева высоты $n k$, представляющего все двоичные наборы этой длины, каждой вершине которого соответствует состояние, в котором не формируются значения выхода. Это дерево будет служить для определения, какому из полуинтервалов принадлежит двоичное число, соответствующее входному двоичному набору. Первому из полуинтервалов соответствуют ветви $0,0,0,0, \ldots, 0,0$ и $0,0,0,0, \ldots, 0,1$. Каждая последующая ветвь соответствует правым концам полуинтервалов. При этом дополнительно появляются $\left\lfloor\log \left((i+1)^{n}-i^{n}\right)\right\rfloor$ новых состояний. Все переходы в промежутке между двумя ветвями представляют собой двоичные наборы, относящиеся к соответствующему интервалу, и направлены на первое состояние конечного автомата, генерирующее нужную выходную последовательность. Таким образом, часть конечного автомата не формирующая выходные значения, содержит $\sum_{i=0}^{2^{k}-1}\left\lfloor\log \left((i+1)^{n}-i^{n}\right)\right\rfloor$ состояний, а обычные конечные автоматы имеют $k 2^{k}$ состояний. Теорема доказана.

Теорема 3. Число автоматов с задержкой с $r$ состояниями не превосходит $(3 r)^{r 2^{n}}$.

Доказательство этого утверждения легко получается из вывода оценки числа конечных автоматов с фиксированным числом состояний [8].

В [6] и [7] для каждого из рассматриваемых в дальнейшем классов была предложена конструкция, позволяющая реализовывать функции с помощью детерминированных 
функций с задержкой (константные функции). Они устроены так: задержка реализуется полным двоичным деревом, высота которого равна задержке, а с каждой ветвью дерева связан конечный автомат, порождающий $k$ разрядов приближенного значения функции. Эта конструкция, по существу, совпадает с использованной при доказательстве теоремы 1.

Теорема 4. Если непрерывная функция одного аргумента $f(x)$ может быть є-приближена, $\varepsilon=1 / 2^{k}$, детерминированной функцией с задержкой $\tau$, то она может быть $\varepsilon$-приближена конечным автоматом с той же задержкой и числом состояний, равным $2^{\tau+1}+k 2^{\tau}$.

Доказательство. Разобьем отрезок $[0,1]$ на полуинтервалы точками $0,1 / 2^{\tau}, 2 / 2^{\tau}, 3 / 2^{\tau}$, $4 / 2^{\tau}, \ldots, i 2^{\tau},(i+1) / 2^{\tau}, \ldots,\left(2^{\tau}-1\right) / 2^{\tau}, 1$. Так как $f(x)$ может быть $\varepsilon$-приближена, $\varepsilon=1 / 2^{k}$, детерминированной функцией с задержкой $\tau$, то изменение функции $f(x)$ на каждом из полуинтервалов $\left[i / 2^{\tau},(i+1) / 2^{\tau}\right)$ не превосходит $1 / 2^{k}$. Поэтому, если в качестве приближенного значения этой функции взять $k$ первых разрядов числа $f(y)$, где $y$ - середина этого полуинтервала, то это значение будет отличаться от значения этой функции в любой другой точке полуинтервала менее, чем на $1 / 2^{k}$. Построим конечный автомат с задержкой $\tau, \varepsilon$-приближающий эту функцию. Автомат состоит из части двоичного дерева высоты $\tau$, представляющего все двоичные наборы этой длины, каждой вершине которого соответствует состояние, в котором не формируются значения выхода. Это дерево будет служить для определения, какому из полуинтервалов принадлежит двоичное число, соответствующее входному двоичному набору. Первому из полуинтервалов соответствуют ветви $0,0,0,0, \ldots, 0,0$ и $0,0,0,0, \ldots, 0,1$. Каждая последующая ветвь соответствует правым концам полуинтервалов. При этом появляются $2^{\tau+1}$ состояний. Все висячие вершины дерева направлены на первое состояние конечного автомата, генерирующего нужную выходную последовательность. Таким образом, часть конечного автомата, не формирующая выходные значения, содержит $2^{\tau+1}$ состояний, а обычные конечные автоматы имеют $k 2^{\tau}$ состояний. Теорема доказана.

Укажем верхние и нижние оценки числа состояний конечного автомата с задержкой для некоторых классов функций, для которых известны оценки числа функций в $2 \varepsilon$-различимом множестве (см. $[1,3])$. Напомним, предполагается, что $\varepsilon=1 / 2^{k}$.

\section{1. Класс функций, удовлетворяющих условию Гельдера}

Напомним, что функция $f(\tilde{x})$ удовлетворяет условию Гельдера с показателем $\alpha$ и константой $L$, если для любых $\tilde{x}, \tilde{y}$

$$
|f(\tilde{x})-f(\tilde{y})| \leqslant L\|\tilde{x}-\tilde{y}\|^{\alpha},
$$

где в качестве нормы рассматривается максимум модуля разности координат. В дальнейшем будем рассматривать именно такую норму и предполагать, что $L=1$; это не ограничивает общность рассмотрения. Обозначим $G_{n}(\alpha)$ класс функций не более чем от $n$ аргументов, удовлетворяющих условию Гельдера с показателем $\alpha$ и константой $L=1$.

Теорема 5. Для некоторой константы С справедливы оценки

$$
\begin{aligned}
& L\left(G_{1}(1), \varepsilon\right) \leqslant 2^{(k+1) / \alpha}, \\
& L\left(G_{1}(1), \varepsilon\right) \geqslant C 2^{k / 2} .
\end{aligned}
$$


Доказательство. В [6] доказано, что при $\varepsilon=1 / 2^{k}$ любую функцию класса $G_{n}(\alpha)$ можно приблизить детерминированной функцией с задержкой $\tau \leqslant(k+1) / \alpha$. Из этого результата и теоремы 3 получается верхняя оценка $L\left(G_{1}(1), \varepsilon\right) \leqslant 2^{(k+1) / \alpha}$.

Для получения нижней оценки воспользуемся результатом работ $[1,3]$ о том, что в классе $G_{1}(1)$ существует $2 \varepsilon$-различимое множество функций порядка $2^{C / \varepsilon}$ при некоторой константе $C$. Так как число автоматов с задержкой, $\varepsilon$-приближающих эти функции, не может быть меньше этого числа, то из теоремы 2 получаем неравенство

$$
(3 r)^{2 r} \geqslant 2^{C 2^{k}},
$$

где $r$ - число состояний автомата и $\varepsilon=1 / 2^{k}$. Из этого неравенства получается нижняя оценка числа состояний.

\section{2. Класс $F_{m}$}

Класс $F_{m}$ определяется как класс непрерывных на отрезке $[0,1]$ функций, имеющих $m$ производных. Функции $f \in F_{m}$ и их производные удовлетворяют неравенствам

$$
\max \left|f^{(i)}(x)\right| \leqslant 1, \quad i=1,2, \ldots, m,
$$

где максимум берется на отрезке $[0,1]$. При этом выполнены условия

$$
f^{(i)}(0)=0, \quad i=1,2, \ldots, m-1 .
$$

Теорема 6. Для некоторой константы С справедливы оценки

$$
\begin{aligned}
L\left(F_{m}, \varepsilon\right) & \leqslant 2^{k+1}(k+2), \\
\log L\left(F_{m}, \varepsilon\right) & \geqslant C \frac{k}{2 m},
\end{aligned}
$$

где C - некоторая константа и логарифм берется по основанию 2.

Доказательство. В [6] доказано, что при $\varepsilon=1 / 2^{k}$ любую функцию класса $F_{m}$ можно приблизить детерминированной функцией с задержкой $\tau \leqslant k+1$. Из этого результата и теоремы 3 получается верхняя оценка $L\left(F_{m}, \varepsilon\right) \leqslant 2^{k+1}(k+2)$.

Для получения нижней оценки заметим, что в $F_{m}$ существует $2 \varepsilon$-различимое множество функций этого класса, содержащее порядка $2^{C(1 / \varepsilon)^{1 / m}}$ функций $($ см. $[1,3])$. Число автоматов с задержкой, $\varepsilon$-приближающих эти функции, не может быть меньше этого числа, поэтому из теоремы 2 получаем неравенство

$$
(3 r)^{2 r} \geqslant 2^{C\left(2^{k}\right)^{1 /(2 m)}},
$$

где $r$ - число состояний автомата и $\varepsilon=1 / 2^{k}$. Из этого неравенства получается нижняя оценка числа состояний.

\section{3. Класс функций от $n$ аргументов, удовлетворяющих условию Гельдера}

Пусть $E^{n}-n$-мерное евклидово пространство и $I^{n}$ есть $n$-мерный замкнутый куб, задаваемый неравенствами $0 \leqslant x_{i} \leqslant 1, i=1,2, \ldots, n$. Рассмотрим множество $F_{m} G_{n}(\alpha)$ действительных функций $n$ переменных, определенных на $I^{n}$, все частные производные 
которых порядка не выше $m$ удовлетворяют условию Гельдера с показателем $0<\alpha \leqslant 1$ и константой $L=1$, и таких, что

$$
\left|\frac{\partial^{k_{1}+k_{2}+\ldots+k_{n}}}{\partial_{x_{1}}^{k_{1}} \partial_{x_{2}}^{k_{2}} \ldots \partial_{x_{n}}^{k_{n}}} f(0,0, \ldots, 0)\right| \leqslant 1
$$

при условии

$$
\sum_{i=1}^{n} k_{i} \leqslant m
$$

Теорема 7. Для некоторой константы $C$ справедливы оиченки

$$
\begin{aligned}
& L\left(F_{m} G_{n}(\alpha), \varepsilon\right) \leqslant 2^{(k+1) / \alpha}, \\
& L\left(F_{m} G_{n}(\alpha), \varepsilon\right) \geqslant C 2^{(n / 2)(k /(m+\alpha-1)} .
\end{aligned}
$$

Доказательство. В [6] доказано, что при $\varepsilon=1 / 2^{k}$ любую функцию класса $F_{m} G_{n}(\alpha)$ можно приблизить детерминированной функцией с задержкой $\tau \leqslant(k+1) / \alpha$.Из этого результата и теоремы 3 получается верхняя оценка $L\left(F_{m} G_{n}(\alpha), \varepsilon\right) \leqslant 2^{(k+1) / \alpha}$.

Для получения нижней оценки заметим, что в классе $F_{m} G_{n}(\alpha)$ существует $2 \varepsilon$-различимое множество функций этого класса, содержащее порядка $2^{C / \varepsilon^{n /(m+\alpha)}}$ функций (см. $[1,3])$. Число автоматов с задержкой, $\varepsilon$-приближающих эти функции, не может быть меньше этого числа, поэтому из теоремы 2 получаем неравенство

$$
(3 r)^{r 2^{n}} \geqslant 2^{C / \varepsilon^{n /(m+\alpha)}},
$$

где $r$ - число состояний автомата и $\varepsilon=1 / 2^{k}$. Из этого неравенства получается нижняя оценка числа состояний.

\section{4. Класс $F_{A}$ аналитических функций}

Класс $F_{A}$ определяется как класс аналитических в некоторой области функций, производные которых удовлетворяют неравенствам

$$
\max \left|f^{(i)}(x) / i !\right| \leqslant 1 / 2^{i}, \quad i=1,2, \ldots
$$

Теорема 8. Для некоторой константы С справедливы оценки

$$
\begin{aligned}
& L\left(F_{A}, \varepsilon\right) \leqslant 2^{k+1}(k+2), \\
& L\left(F_{A}, \varepsilon\right) \geqslant C k .
\end{aligned}
$$

Доказательство. В работе [6] доказано, что при $\varepsilon=1 / 2^{k}$ любую функцию класса $F_{A}$ можно приблизить детерминированной функцией с задержкой $\tau \leqslant k+1$. Из этого результата и теоремы 3 получается верхняя оценка $L\left(G_{1}(1), \varepsilon\right) \leqslant 2^{k+1}(k+2)$.

Для получения нижней оценки воспользуемся результатом работ $[1,3]$ о том, что в классе $F_{A}$ существует $2 \varepsilon$-различимое множество функций порядка $2^{C \log ^{2}(1 / \varepsilon)}$ при некоторой константе $C$. Так как число автоматов с задержкой, $\varepsilon$-приближающих эти функции, не может быть меньше этого числа, из теоремы 2 получаем неравенство

$$
(3 r)^{r 2^{n}} \geqslant 2^{C \log ^{2}(1 / \varepsilon)}
$$

где $r$ - число состояний автомата и $\varepsilon=1 / 2^{k}$. Из этого неравенства получается нижняя оценка числа состояний. 
В заключение автор выражает свою благодарность В. А. Буевичу за постановку задачи и существенную помощь при оформлении полученных результатов.

\section{Список литературы}

1. Витушкин А. Г., Оценка сложности задачи табулящии. Физматгиз, Москва, 1959.

2. Гашков С. Б., О сложности приближенной реализации непрерывных функций схемами и формулами в полиномиальных и некоторых других базисах. Математические вопросы кибернетики (1994) №5, 144-207.

3. Офман Ю. П., О приближенной реализации непрерывных функций на автоматах. Доклады АН ССCP (1963) 152, №4, 823-825.

4. Черепов А. Н., Оценки сложности приближения непрерывных функций некоторых классов детерминированными функциями с задержкой.. В сб.: Труды 16 Международной школь-семинара «Синтез и сложность управляющих систем». Мех.-мат. ф-т МГУ, Москва, 2006, с. 118-122.

5. Черепов А. Н., О сложности приближения непрерывных функций детерминированными функциями с задержкой. В сб.: Материалы 9 Международной конференции «Интеллектуальные системы и компьютерные науки». Мех.-мат. ф-т МГУ, Москва, 2006, с. 307-310.

6. Черепов А. Н., Оценки сложности приближения непрерывных функций некоторых классов детерминированными функциями с задержкой. Дискретная математика (2008) 20, №4, 147156.

7. Черепов А. Н., О приближении непрерывных функций детерминированными функциями с задержкой. Дискретная математика (2010) 22, №1, 83-103.

8. Яблонский С. В., Введение в дискретную математику. Наука, Москва, 1979.

Статья поступила 11.11.2011. 